\title{
Neridronato nel trattamento dell'osteogenesi imperfetta: prestazioni cliniche ed economiche di un farmaco orfano
}

Orietta Zaniolo*, Mario Eandi*

\begin{abstract}
Bisphosphonates (BPs) are osteoclast-mediated bone resorption inhibitors and the nature of the groups attached to the central carbon atom determines the drug potency. Neridronate is an injectable aminobisphosphonate, structurally similar to alendronate and pamidronate, authorized for the treatment of osteogenesis imperfecta (OI).

This drug has often been used to treat other pathologies, as an off-label option, to increase tolerability and ameliorate compliance, partly because the management of orally administered bisphosphonates, with their gastrointestinal side effects, results complicated.
\end{abstract}

In this paper pharmacokinetic, pharmacodinamic and main placebo-controlled clinical trials on OI patients are reviewed. The available scientific evidence demonstrates the neridronate efficacy to improve spine and hip bone mineral density, to lower markers of skeletal turnover and to decrease fracture incidence, compared with controls.

We also report clinical trials results and data about the effect of intravenous infusions of neridronate in patients with postmenopausal osteoporosis, Paget's disease and rheumatoid arthritis.

Finally, we consider the economical impact of chronic and incapacitating pathologies, like osteogenesis imperfecta, on family's total income and the influence of the disease on quality of life of pediatric and adult patients.

Farmeconomia e percorsi terapeutici 2004; 5 (3): 163-178

\section{INTRODUZIONE}

I bifosfonati sono analoghi del pirofosfato in cui il ponte $\mathrm{P}-\mathrm{O}-\mathrm{P}$ è stato sostituito con un ponte $\mathrm{P}-\mathrm{C}-\mathrm{P}$, non idrolizzabile. Grazie alla loro particolare attività nel mantenere l'omeostasi minerale ossea, sono attualmente i farmaci più utilizzati nel trattamento dell'osteolisi tumorale con ipercalcemia e del morbo di Paget, oltre che nella prevenzione primaria e secondaria dell'osteoporosi.

Il neridronato, un amino-bifosfonato di recente introduzione, è stato utilizzato con successo in alcuni trial clinici per il trattamento dell'osteoporosi post-menopausale, del morbo di Paget e soprattutto dell'osteogenesi imperfetta $(\mathrm{OI})$, malattia rara e gravissima contro la quale questo farmaco ha mostrato risultati molto incoraggianti.
L'OI è una patologia ereditaria del tessuto connettivo, caratterizzata da fragilità ossea e fratture ricorrenti che, in molti casi, conducono a deformità scheletriche.

La maggior parte dei pazienti con osteogenesi imperfetta presenta una diminuzione della densità ossea anche se a volte, sia negli adulti sia nei bambini, l'aspetto radiologico appare normale. La densitometria ossea a doppio raggio-X conferma la diminuzione della densità dell'osso sia trabecolare (vertebre, coste) sia corticale (omero, femore), soprattutto in caso di gravidanza [1], dopo la menopausa e, negli uomini, dopo i 50 anni [2].

Pur trattandosi di una patologia eterogenea in termini sia di ereditarietà (dominante, recessiva, sporadica/nuova mutazione) sia di espressività fenotipica, gli individui affetti da
*Farmacologia clinica, Università di Torino 
OI comunemente presentano bassa statura, sclere blu, dentinogenesi imperfetta, perdita di udito nell'età adulta, scoliosi e lassità legamentosa.

Generalmente, sulla base delle caratteristiche cliniche e genetiche, si distinguono quattro tipi di OI (Tabella 1).

I soggetti affetti dalle forme più gravi presentano fratture multiple sin dalla nascita e sviluppano precocemente deformazioni scheletriche: il decesso nella grande maggioranza dei casi avviene nelle prime ore o nei primi giorni di vita; la sopravvivenza oltre l'anno di vita è rara. Nelle forme severe (tipo III e IV) c'è sempre il rischio di complicazioni cardio-polmonari, dovute a deformità spinali e toraciche, che possono portare all'exitus durante l'infanzia [3]. Il numero e l'età di inizio delle fratture e delle deformità hanno un ruolo nel determinare il raggiungimento della capacità di camminare e quindi la futura autonomia del bambino [4].

La causa principale di questa patologia è da ricercare nella mutazione dei geni del collagene di tipo I [6]; non sono state rilevate, al momento, particolari correlazioni tra i fenotipi clinici e specifiche mutazioni, anche se sembra che quelle localizzate nella parte $\mathrm{N}$-terminale dell'elica del collagene siano associate a fenotipi meno gravi, mentre quelle della parte C-terminale sarebbero associate a forme di malattia più gravi.

Nei casi più lievi le deformità scheletriche non sono costanti, a dispetto delle fratture multiple, e l'altezza dei pazienti può essere quasi normale [5].

In questo caso, la capacità motoria non è ridotta in maniera grave, per cui spesso è mantenuta la possibilità di deambulare e di una vita autonoma. Invece quasi tutti i pazienti con OI tipo III e una buona parte dei pazienti con tipo IV sono costretti sulla sedia a rotelle, malgrado gli ausili e una riabilitazione intensiva.

\section{PROFILO FARMACOLOGICO CLINICO DEL NERIDRONATO}

\section{Farmacodinamica}

Il neridronato appartiene alla classe dei bifosfonati, composti che agiscono sul metabolismo osseo impedendo il riassorbimento, con conseguente diminuzione del turnover osseo.

La struttura chimica $\mathrm{P}-\mathrm{C}-\mathrm{P}$ possiede grande affinità per il $\mathrm{Ca}++$ (base della selettività per l'osso di questi composti) ed è molto resistente all'idrolisi in ambiente acido e all' azione delle pirofosfatasi: caratteristiche fondamentali per l'azione farmacologica.

Gli effetti scheletrici dei bifosfonati sono mediati da un'azione inibitoria diretta sul reclutamento, la differenziazione, l'attività e il ciclo vitale degli osteoclasti, nonché da un'azione indiretta sugli osteoblasti, in cui induce una diminuzione nel rilascio dei mediatori che in situazioni normali attivano gli osteoclasti. Ciò determina una rapida inibizione dell' attività osteoclastica e un effetto, più ritardato nel tempo, sulla deposizione osteoblastica [7-9]. Nella maggior parte dei casi, si ottiene un sostanziale incremento della densità minerale ossea [10].

Il meccanismo con cui questa classe di farmaci esplica i propri effetti non è completamente chiaro, ma pare che essi rallentino la formazione e la dissoluzione dei cristalli di idrossiapatite mimando l'azione del loro analogo naturale, il pirofosfato. In particolare, l'elevata affinità in vivo della struttura $\mathrm{P}-\mathrm{C}-\mathrm{P}$ per l'idrossiapatite induce la deposizione dei bifosfonati nella matrice ossea [11-13].

Si ipotizza che una volta incorporati nella matrice ossea, i bifosfonati siano inglobati durante i processi di riassorbimento osseo dagli osteoclasti maturi mediante endocitosi, inibendo la loro attività mediante il blocco della produzione di enzimi litici, delle secrezioni acide e

\begin{tabular}{ll}
\hline Classificazione & \\
\hline Tipo I & $\begin{array}{l}\text { Forma più frequente e meno grave, caratterizzata da un deficit quantitativo (circa } 50 \% \text { ) del collagene, sclere } \\
\text { blu, aumentata incidenza di fratture nell'infanzia e nell'età avanzata. Spesso misconosciuta }\end{array}$ \\
Tipo II & È la forma più grave con morte perinatale \\
Tipo III & $\begin{array}{l}\text { Forma severa con bassa statura, gravi deformità scheletriche che condizionano qualità e quantità di vita dei } \\
\text { pazienti, sclere blu e dentinogenesi imperfetta }\end{array}$ \\
Tipo IV & Forma intermedia tra il tipo I e III con statura piuttosto bassa e modeste deformità scheletriche \\
\hline
\end{tabular}

Tabella 1

Classificazione dell'osteogenesi imperfetta (generalmente, sulla base delle caratteristiche cliniche e genetiche, si distinguono quattro tipi di Ol) 
delle prostaglandine. Inoltre essi indurrebbero negli osteoclasti alterazioni morfologiche apoptotiche (retrazione, condensazione nucleare, frammentazione cellulare) e biochimiche (frammentazione del DNA e attivazione delle caspasi), riducendo la durata media della vita di queste cellule e i correlati processi di riassorbimento.

Un'altra possibilità è che il bifosfonato accumulato all'interno dell'osso venga nel tempo rilasciato in piccole quantità alle circostanti aree di elevato turnover; ciò spiegherebbe il fatto che, nonostante i bassi livelli di farmaco attivo circolante, una singola somministrazione spesso sia sufficiente a mantenere una sostanziale inibizione del riassorbimento osseo anche per alcuni anni.

L'azione indiretta svolta dai bifosfonati si esplica a livello degli osteoblasti, i quali, in condizioni fisiologiche, attivano, mediante un legante presente sulla loro membrana (RANKL), un recettore espresso sulla membrana del precursore emopoietico dell'osteoclasta (RANK) inducendo la sua differenziazione e maturazione a osteoclasta attivo. Il farmaco probabilmente interferisce con questo meccanismo, inducendo la produzione di osteoprotegerina (OPG) da parte degli osteoblasti. L'OPG è una proteina naturale che, legandosi essa stessa al RANKL, impedisce l'azione del ligando sul RANK e la conseguente osteoclastogenesi [14,15].

Analizzando le curve dose-risposta relative al neridronato e agli altri bifosfonati è possibile osservare che anche basse concentrazioni di farmaco sono in grado di indurre gli effetti clinici desiderati; per esempio osteoblasti esposti per soli 5 minuti a una modestissima dose di bifosfonato sono stati in grado di inibire la maturazione osteoclastica $[16,17]$. Tali considerazioni suggeriscono l'esistenza di un recettore o di un sito di legame cellulare specifico per questa classe di farmaci, anche se a oggi esso non è stato ancora identificato.

Studi in vitro e in vivo hanno dimostrato che il trattamento con neridronato comporta riduzioni significative dei parametri di riassorbimento e della fosfatasi alcalina indice di turnover osseo, senza però modificare il processo di mineralizzazione. I marker biochimici del riassorbimento subiscono decrementi molto rapidi (pochi giorni nel caso di somministrazione parenterale) anticipando di alcune settimane la riduzione della fosfatasi alcalina, che mostra un andamento più lento e graduale. La rapida inibizione del riassorbimento osseo provoca un transitorio disaccoppiamento tra i processi di neoformazione e quelli di riassorbimento; questo stato perdura sino a quando non venga raggiunto un nuovo equilibrio, con l'adeguamento della neosintesi ossea ad un livello di turnover più basso. La prevalenza dei processi di osteosintesi durante la fase di disaccoppiamento determina la riduzione dell'afflusso di calcio verso l'ambiente extracellulare e un temporaneo aumento della secrezione di paratormone. L'insorgenza di un iperparatiroidismo secondario permette di contrastare l'ipocalcemia incrementando la sintesi di calcitriolo e stimolando l'assorbimento intestinale di calcio. Inoltre l'aumento del paratormone diminuisce il riassorbimento tubulare dei fosfati, diminuendone in tal modo la concentrazione sierica [18].

\section{FARMACOCINETICA}

I bifosfonati hanno una bassissima biodisponibilità orale, peraltro condizionata dalla contemporanea assunzione di cibo o farmaci: dopo somministrazione per via orale solo una minima quantità di bifosfonato viene assorbita (biodisponibilità orale inferiore all' $1 \%$ per i bifosfonati azotati). Questo è parzialmente spiegato dalla loro bassa lipofilia, dalla elevata carica negativa e dalla forma insolubile con cui si presentano a livello intestinale a causa del legame con il calcio.

Il contatto di questi farmaci con la mucosa dell'apparato digerente può provocare fenomeni irritativi $[19,20]$ : risulta quindi importante assumere i bifosfonati con abbondante acqua demineralizzata calda, a completo digiuno (sia prima che dopo) e stando in piedi per almeno trenta minuti [21].

Al contrario, le formulazioni parenterali, come quelle con cui viene somministrato il neridronato, sono quelle che garantiscono una piena biodisponibilità del principio attivo nel caso di somministrazione endovenosa e, spesso, anche intramuscolare.

Le caratteristiche farmacocinetiche del sodio neridronato sono state valutate nel plasma e nelle urine di ratto dopo somministrazione endovenosa singola di $10 \mathrm{mg} / \mathrm{kg}$. I risultati dell' analisi hanno dimostrato una concentrazione massima $(0,06 \mathrm{mg} / \mathrm{ml})$ raggiunta subito dopo la somministrazione, seguita da una fase rapida di riduzione delle concentrazioni plasmatiche, dovuta ai fenomeni di distribuzione tissutale e di eliminazione; nello studio il $31,7 \%$ del farmaco veniva escreto nelle urine durante le 24 ore successive alla somministrazione.

Nell'uomo l'infusione endovenosa lenta di 25,50 e 100 mg ha mostrato una chiara linearità e proporzionalità fra le dosi; con la somministrazione intramuscolare, i profili di escre- 


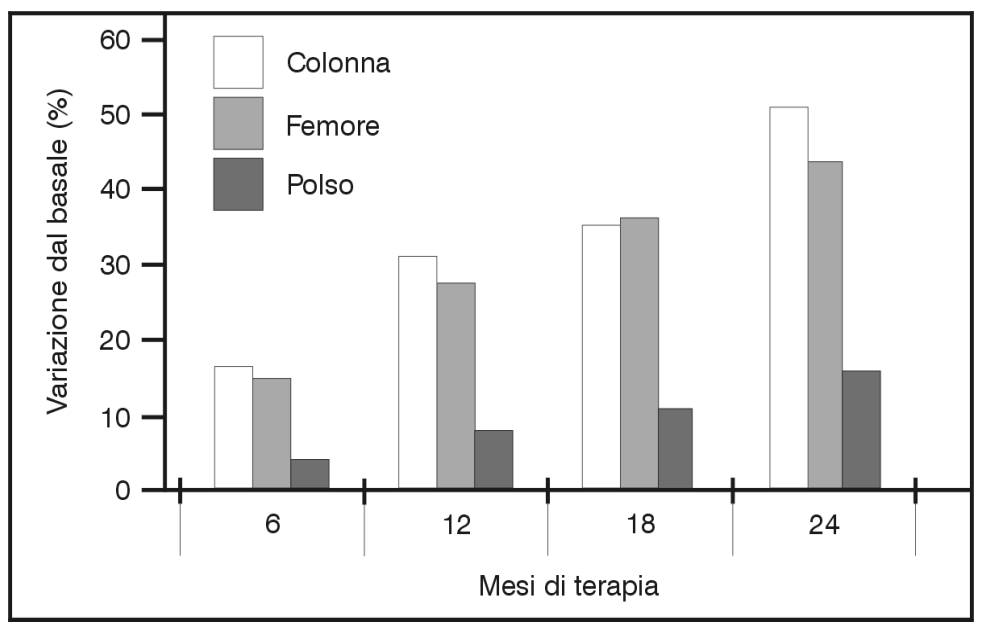

Figura 1

Variazioni densitometriche in corso di terapia con neridronato in soggetti di età inferiore a 20 anni [26]

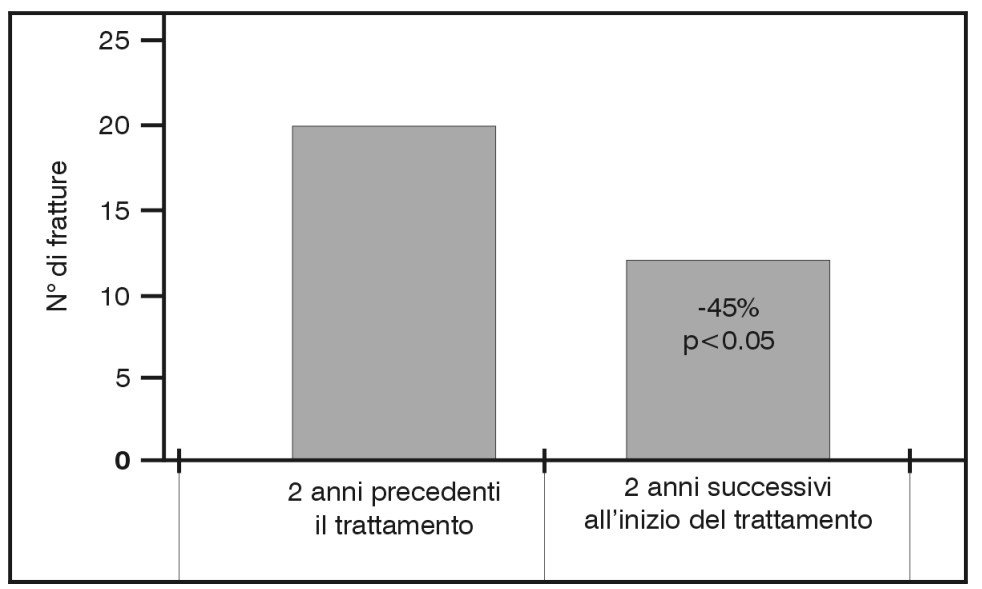

Figura 2

Confronto tra il numero di eventi fratturativi verificatisi nei due anni precedenti il trattamento e durante $i$ due anni di terapia con neridronato nel gruppo pediatrico [26] una zona piuttosto che un'altra dipenda fondamentalmente dalla quantità di farmaco somministrata: modeste quantità favoriscono l'accumulo nelle aree di riassorbimento, mentre elevate quantità si distribuiscono abbastanza equamente nei due diversi tipi di siti.

Solitamente i bifosfonati non si accumulano nei tessuti molli, tuttavia si può verificare un deposito a livello di stomaco, fegato e milza in particolari condizioni in cui, a causa di una somministrazione endovenosa troppo elevata o troppo rapida, si possono formare grossi complessi con il ferro e con il calcio, fagocitati poi dai macrofagi del sistema reticoloendoteliale. Sempre a causa della formazione di questi aggregati insolubili, infusioni troppo rapide e dosi troppo alte possono risultare dannose anche a livello renale [22].

L'emivita di eliminazione è di circa 7 ore e, circa la metà della dose somministrata per infusione endovenosa o per via intramuscolare, viene escreta nelle urine dopo ogni somministrazione. Il farmaco in vivo non è metabolizzato, probabilmente a causa dell' elevata stabilità del legame $\mathrm{P}-\mathrm{C}-\mathrm{P}$ nei confronti degli enzimi idrolitici: esso viene escreto pressoché inalterato.

L'eliminazione di questi farmaci è molto lenta, infatti essi permangono a lungo a livello scheletrico; è stata calcolata un' emivita nello scheletro di diversi anni [23].

Non sono disponibili dati che rilevino il comportamento farmacocinetico del farmaco in soggetti con insufficienza renale o epatica.

\section{Efficacia terapeutica}

Sono oggi disponibili i dati relativi al trattamento dell'osteogenesi imperfetta con neridronato sia nei pazienti in età pediatrica che in quelli adulti $[24,25]$.

81 pazienti di ambo i sessi, 52 sopra i 20 anni (range 21-71) e 29 di età inferiore (range 517), in gran parte affetti dalla malattia nella forma più lieve, ma con qualche caso di media (tipo IV=20) ed elevata (tipo III=10) gravità [26] sono stati trattati con un protocollo terapeutico che prevedeva la somministrazione trimestrale di una dose di neridronato compresa fra un minimo di $2 \mathrm{mg} / \mathrm{kg}$ e un massimo di $100 \mathrm{mg} / \mathrm{kg}$.

Nel gruppo pediatrico, in cui la dose somministrata è stata di $2 \mathrm{mg} / \mathrm{kg}$ di peso corporeo, si sono osservati i maggiori incrementi di massa ossea, accompagnati da significativi miglioramenti a livello delle vertebre e di tutti i distretti studiati. Gli effetti, evidenti già dopo 6 mesi di terapia, sono stati osservati per tutta la durata del trattamento (Figura 1). 
Sempre nel gruppo pediatrico, tra i due anni precedenti l'inizio del trattamento e i due anni di terapia si è verificato un calo di circa il $50 \%$ dell'incidenza di frattura (Figura 2).

Oltre al miglioramento di tali parametri oggettivi, i pazienti del gruppo pediatrico hanno riportato anche una notevole attenuazione dei dolori ossei, caratteristici della patologia, e un incremento della mobilità generale: aspetti da non trascurare in vista del considerevole impatto che hanno sulla qualità della vita dei pazienti.

Il protocollo clinico che prevede l'utilizzo del neridronato nel trattamento dei bambini affetti da OI, anche a partire da pochi mesi di vita, è stato valutato dall'équipe di Finocchiaro della Clinica Pediatrica dell'Università La Sapienza di Roma. In questo studio sono stati analizzati gli aminoacidi urinari, indici di metabolismo osseo come l'idrossiprolina e tutta una serie di altre molecole urinarie come il calcio, il fosfato, la fosfatasi alcalina, l'osteocalcina, la taurina, la prolina e il C-telopeptide/creatinina.

Dopo due cicli di somministrazione è stato osservato un aumento del calcio sierico e una diminuzione del fosfato sierico, della fosfatasi alcalina e dell'escrezione del calcio urinario [27].

Per quanto riguarda il trattamento di soggetti adulti sono disponibili i dati relativi ad uno studio controllato e randomizzato svolto da Adami e colleghi (università di Verona), in collaborazione con l'Associazione Italiana Osteogenesi Imperfetta.

I pazienti sono stati randomizzati a ricevere trimestralmente $100 \mathrm{mg}$ di neridronato ev (in $250 \mathrm{ml}$ di fisiologica infusi in 30 minuti) o placebo, con un rapporto di 2:1.

L'apporto dietetico di calcio è stato valutato regolarmente e mantenuto al di sopra di 1.000 mg giornalieri in tutti i pazienti. Supplementi di vitamina $\mathrm{D}_{2}$ sono stati somministrati se i livelli ematici di $25 \mathrm{OH}$-vitamina D scendevano al di sotto di $20 \mathrm{ng} / \mathrm{ml}$ : 5 pazienti hanno ricevuto supplementi di calcio o di vitamina $\mathrm{D}_{2}$.

Dopo i primi 12 mesi di follow up, anche i pazienti del gruppo di controllo hanno iniziato la terapia con neridronato.

Dei 78 partecipanti al trial, 46 hanno terminato lo studio (dopo due anni di follow up); di questi 31 erano stati randomizzati a ricevere neridronato, mentre i rimanenti 15 pazienti facevano parte del gruppo di controllo [24].

Nel gruppo trattato con neridronato, dopo i primi 12 mesi di trattamento si è determinato un aumento del $3,0 \pm 4,6 \%$ nei valori di densità ossea (BMD) della colonna e del 4,3 $\pm 3,9 \%$ in
* $\mathrm{P}<0.05$ fra i gruppi
\# $\mathrm{P}<0.05$ rispetto al basale
$+\mathrm{P}<0.001$ rispetto al basale
10
Densità minerale ossea del rachide
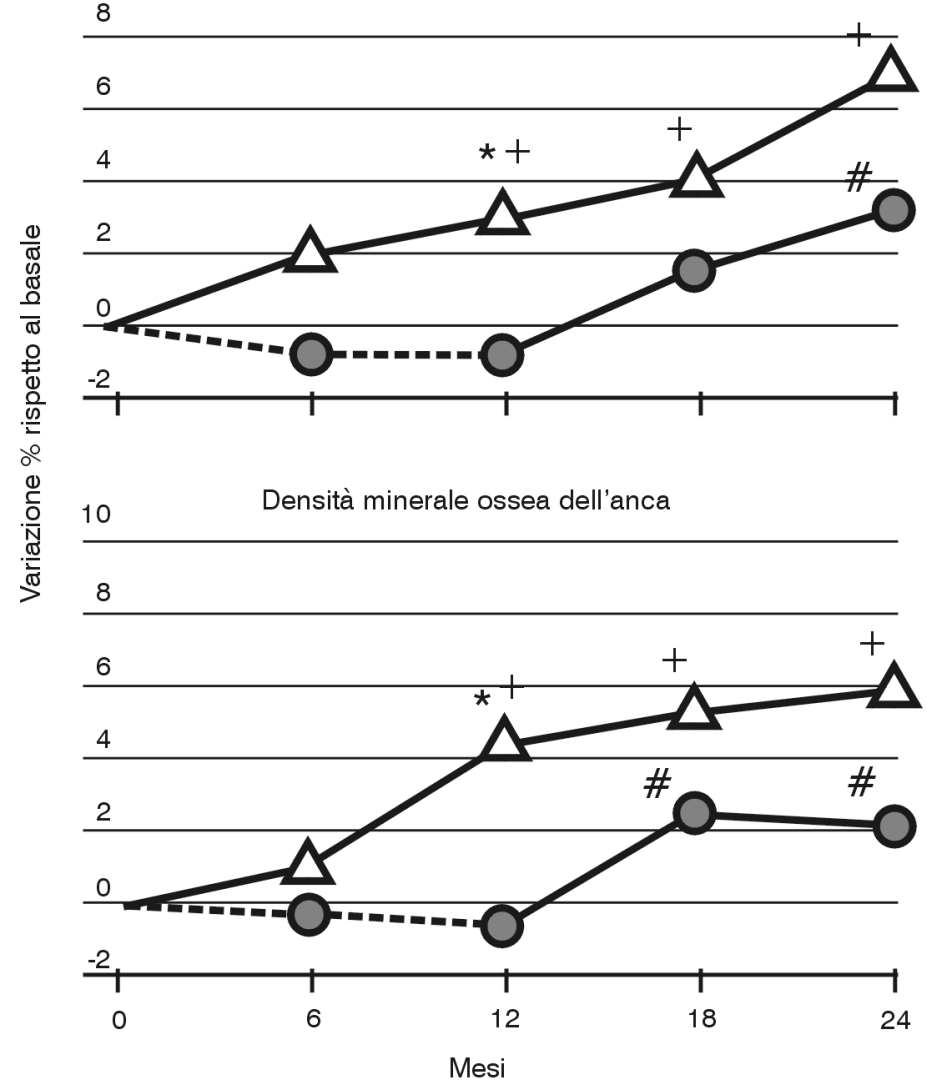

Figura 3

Variazioni della densità minerale ossea durante il trattamento con neridronato ev (linea continua) e durante il periodo di controllo (linea tratteggiata) [24]

quelli dell'anca; valori che, nel corso del secondo anno, sono ulteriormente aumentati rispettivamente al 3,9\% e all' $1,5 \%$.

L'analisi densitometrica dei pazienti del gruppo controllo, che durante il primo anno non ha mostrato variazioni significative, nel corso del secondo anno, e cioè dopo il passaggio alla terapia, ha mostrato valori molto simili a quelli ottenuti il primo anno dal gruppo randomizzato a neridronato (Figura 3).

I valori dei marker di turnover osseo durante il trattamento con neridronato si sono ridotti significativamente; le riduzioni medie sono riportate nei grafici di Figura 4.

Tutti i pazienti mostravano al baseline alcune deformità delle vertebre toraciche e lombari, e in 21 di essi vennero trovate evidenti fratture (schiacciamento delle vertebre). Nei quattro anni precedenti l'inizio dello studio, 16 
* $\mathrm{P}<0.05$ fra i gruppi

\# $P<0.05$ rispetto al basale

$+P<0.01$ rispetto al basale
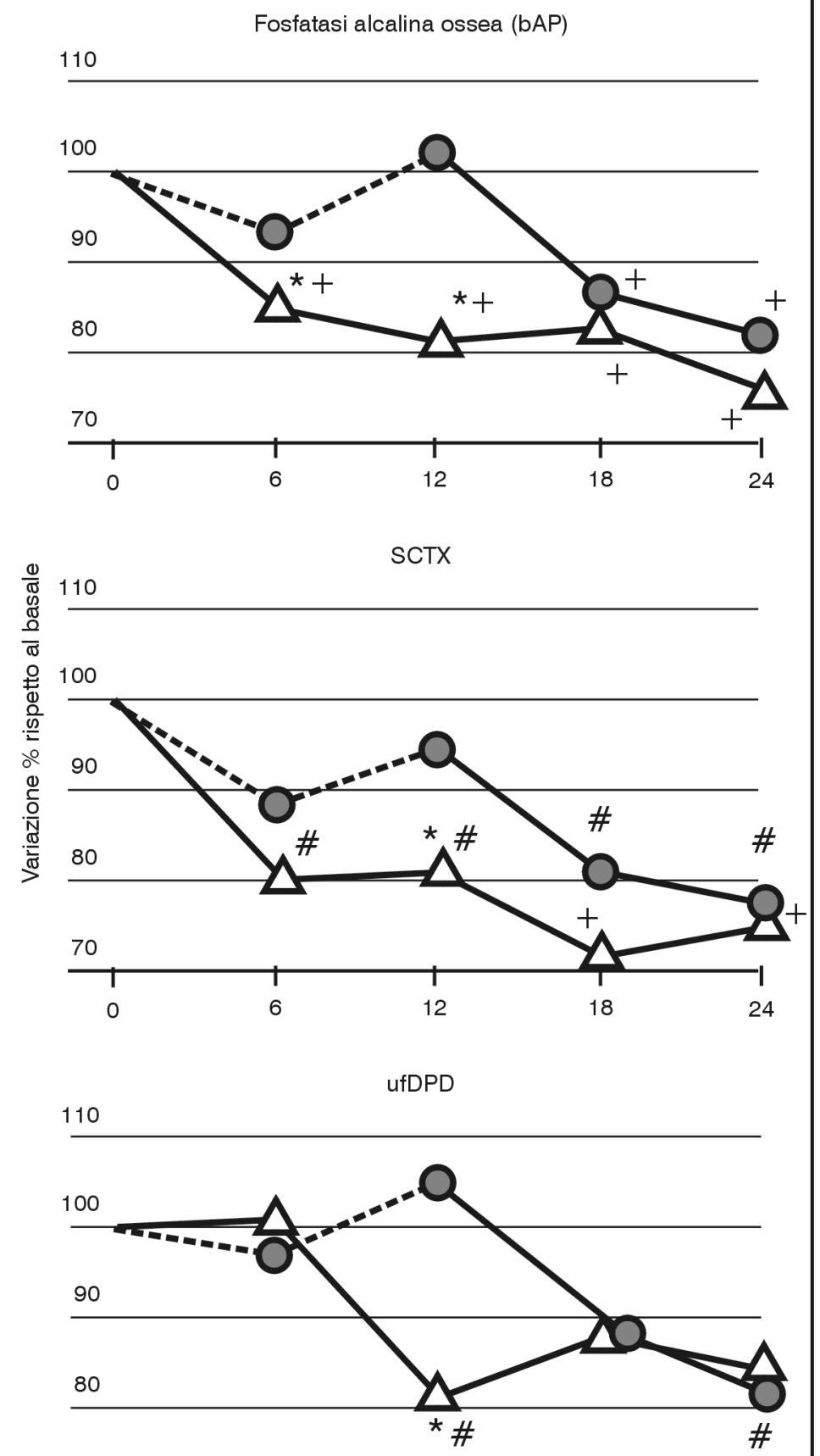

70

\begin{tabular}{ccccc}
70 & & & & \\
\hline 0 & 6 & 12 & 18 & 24 \\
& & Mesi & &
\end{tabular}

Figura 4

Variazioni della fosfatasi alcalina ossea (bAP), del livello sierico di telopeptide C-terminale (SCTX) e del rapporto deossipiridinolina libera urinaria/creatinina (ufDPD) durante il trattamento con neridronato ev (linea continua) e durante il periodo di controllo (linea tratteggiata) [24]

pazienti avevano riportato complessivamente 18 fratture clinicamente evidenti di cui la più recente si era verificata cinque mesi prima dell'arruolamento. Nel gruppo di controllo, una frattura vertebrale e una frattura a un arto si sono verificate durante il primo anno, mentre nessuna durante il secondo anno di follow up. All'interno del gruppo randomizzato a neridronato si è verificata una sola frattura nel corso del trial, una riduzione statisticamente significativa dell'incidenza di fratture rispetto agli anni precedenti lo studio.

Come si evince dai grafici riportati in Figura 3 , nei pazienti che non hanno seguito alcun trattamento non si sono verificate variazioni significative della densità ossea; al contrario, nei pazienti trattati, la massa è aumentata sia rispetto al valore iniziale che al gruppo di controllo, con una crescita confermata anche nel secondo anno di studio. Questi risultati sommati all'aumento della mobilità, all'attenuazione del dolore osseo e, soprattutto, alla diminuzione del rischio di fratture, costituiscono un grosso beneficio per i pazienti affetti dalla patologia.

L'efficacia e la tollerabilità del neridronato sono state valutate anche nella terapia dell'osteoporosi nell'adulto, patologia estremamente diffusa fra le donne nel periodo postmenopausale, ma in progressiva diffusione anche fra i soggetti più giovani, sia donne che uomini. Il possibile utilizzo di un trattamento farmacologico endovenoso intermittente in questa patologia potrebbe aumentare l'adesione alla terapia, rappresentando un considerevole vantaggio per il paziente.

I bifosfonati orali, pur essendo utilizzati con successo nella terapia dell'osteoporosi, sono poco assorbiti dal tratto gastrointestinale e la presenza di cibo nello stomaco abbassa ulteriormente la percentuale di assorbimento; anche la tollerabilità gastrointestinale è molto scarsa, infatti per ridurre gli effetti collaterali il farmaco deve essere ingerito con notevoli quantità d'acqua e mantenendo rigorosamente la posizione eretta per almeno mezz'ora: il che li rende totalmente inaccessibili a coloro che sono costretti a letto, anche solo temporaneamente [28].

Per valutare l'eventuale possibilità di adottare una strategia terapeutica alternativa, basata su neridronato somministrato in modo intermittente per via parenterale, è stato condotto uno studio su 78 donne in menopausa da almeno cinque anni, con un'età non superiore a 80 anni e riduzione della densità ossea del rachide pari ad almeno -2,5 [29]. Il razionale dell'utilizzo dei bifosfonati in maniera intermittente è basato sul fatto che anche una breve inibizione dell' attività osteoclastica lascerà spazio a un periodo di attività osteo- 
blastica incontrastata, con conseguente miglioramento del bilancio osseo. Le pazienti hanno ricevuto per due anni $50 \mathrm{mg}$ ev di neridronato o placebo, in $50 \mathrm{ml}$ di fisiologica ogni 2 mesi; inoltre, entrambi i gruppi hanno ricevuto un supplemento giornaliero di calcio e vitamina $D$ per tutta la durata del trial.

Tutte le pazienti del gruppo neridronato hanno completato lo studio, mentre quattro pazienti del gruppo controllo lo hanno abbandonato per cominciare un trattamento attivo. Nel gruppo neridronato i valori di densità ossea sono cresciuti progressivamente nei due anni di trattamento e queste variazioni si sono mantenute costanti anche durante un ulteriore anno di follow up in cui è stato somministrato solo il supplemento giornaliero di calcio e vitamina D (Figura 5).

Nel gruppo neridronato, inoltre, la fosfatasi alcalina ossea si è ridotta significativamente ($23 \pm 17 \%$ ) nei confronti dei valori basali e del gruppo di controllo entro quattro mesi dall' inizio dello studio, restando soppressa per tutta la durata del trattamento.

I valori sierici di CTX si sono ridotti in modo significativo già due mesi dopo la prima infusione.

Dati clinici comparabili, in termini di incremento della BMD e di riduzione dei marker biochimici, sono stati ottenuti con cicli di somministrazione intramuscolare di neridronato: $25 \mathrm{mg}$ ogni due settimane o $25 \mathrm{mg} /$ die per sei giorni consecutivi ogni tre mesi [30].

In questo studio è stato rilevato un rapido incremento della densità ossea durante i primi sei mesi di terapia, con una tendenza all'incremento ancora evidente alla fine dei due anni di durata dello studio.

Gli studi, di fatto, confermano la sostanziale equivalenza in termini di incremento di massa ossea fra la somministrazione endovenosa e quella intramuscolare. Nonostante ciò, sembrerebbe che il trattamento sia più efficace nel caso in cui venga adottato uno schema posologico più regolare: infatti i risultati in termini di BMD a lungo termine (dopo 24 mesi) sono decisamente migliori [15] nel gruppo randomizzato a ricevere neridronato bisettimanalmente.

Questi studi hanno rilevato che le variazioni del BMD indotte da neridronato $50 \mathrm{mg} / \mathrm{bi}$ mestre sono dello stesso ordine di grandezza di quelle ottenute mediante il trattamento orale con alendronato $10-20 \mathrm{mg} /$ die [31]; queste variazioni appaiono, inoltre, superiori a quelle ottenute con altri aminobifosfonati somministrati con terapia intermittente endovenosa: 4 $\mathrm{mg} / \mathrm{anno}$ di zoledronato o $8 \mathrm{mg} /$ anno di

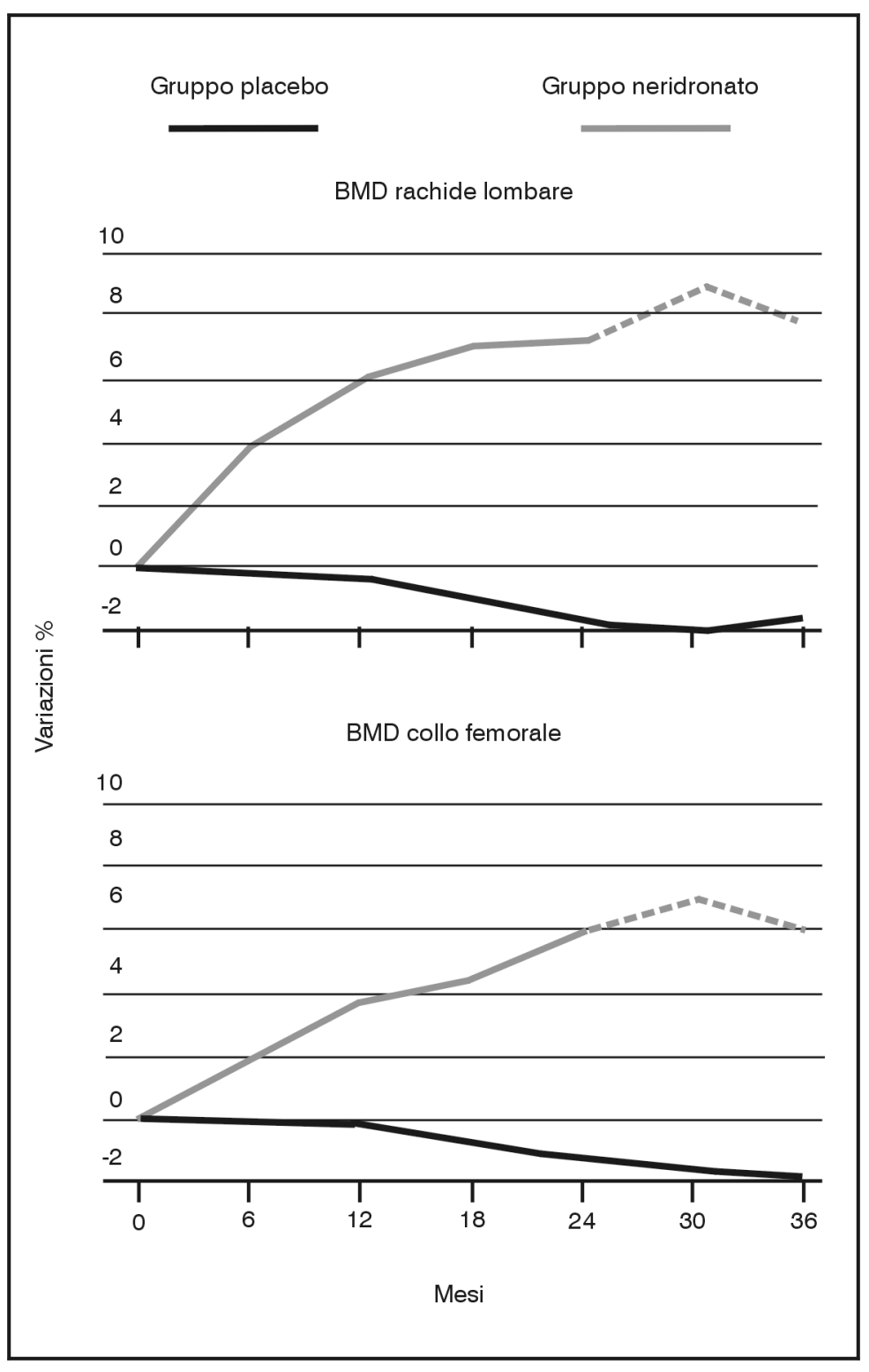

Figura 5

Variazioni \% della BMD del rachide lombare e del femore a 6, 12, 18 e 24 mesi; la linea tratteggiata indica il periodo di follow up [29]

ibandronato aumentano, nei 12 mesi, la BMD del rachide e dell' anca rispettivamente del 5\% e del 1-3\% [32-34], mentre con l'utilizzo del clodronato le variazioni di BMD registrate durante i trial clinici risultano essere ancora più basse [35-38].

Tali risultati collocano il neridronato tra le terapie più valide potenzialmente a disposizione del sistema sanitario. Inoltre, dal momento che l'osteoporosi è una patologia cronica che va trattata per tempi molto lunghi, la collaborazione del paziente riveste un ruolo fondamentale nel raggiungimento degli effetti sperati: alcune tipologie di pazienti (ad esempio allettati o con problemi gastrointestinali) trarrebbero considerevoli benefici da una terapia endovenosa con ampi intervalli fra una somministrazione e l'altra, e ciò ne aumente- 


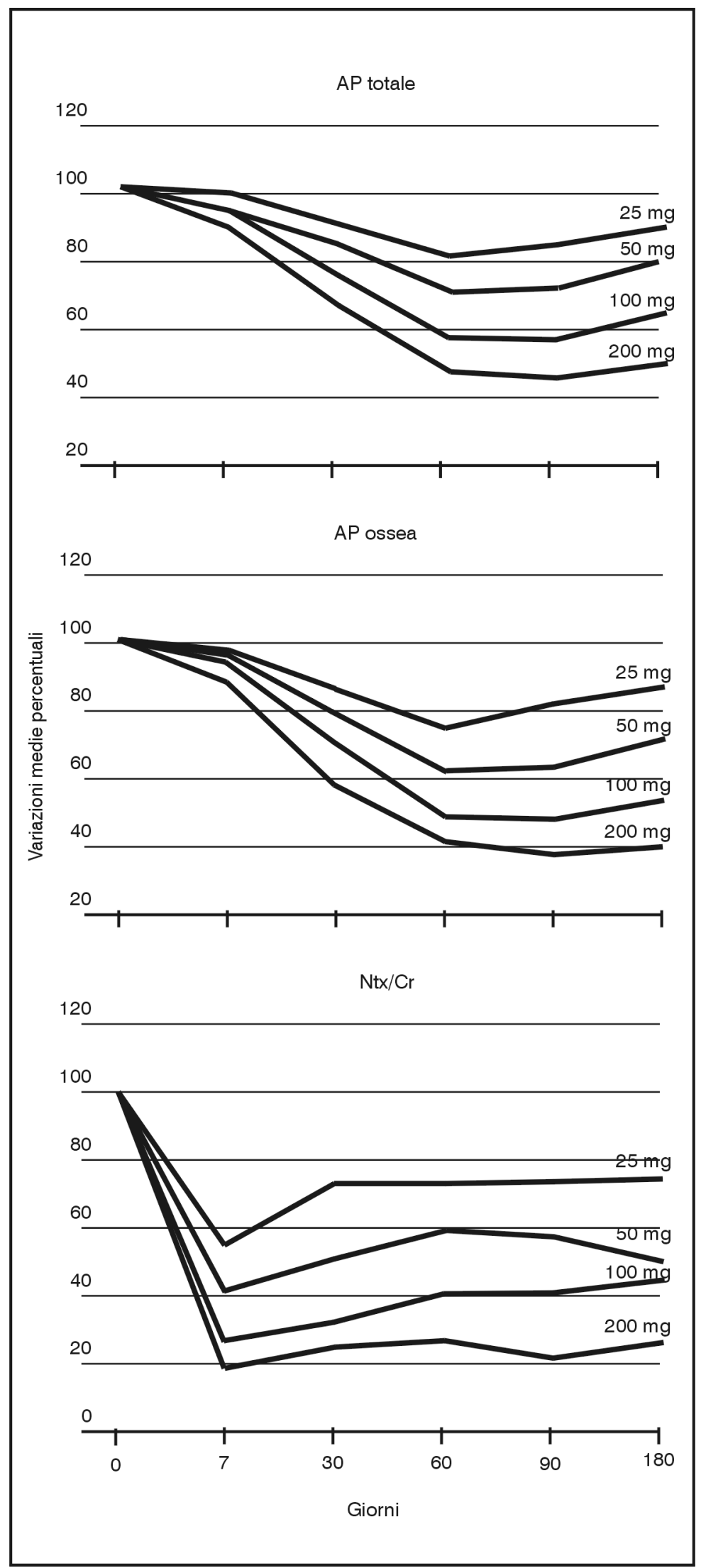

Figura 6

Variazioni medie percentuali rispetto al baseline della fosfatasi alcalina totale (AP totale), ossea (AP ossea) e dell'escrezione di N-telopeptide (NTX/Cr) [41]

rebbe notevolmente la compliance. Il neridronato è risultato essere efficace anche nei confronti di altri disturbi a carico dell'apparato scheletrico, come ad esempio il morbo di Paget. Questa patologia, ad eziologia sconosciuta, è caratterizzata da un aumentato riassorbimento scheletrico con successiva formazione di tessuto osseo patologico a causa del netto incremento della componente vascolare e fibrosa; secondo alcuni studi radiografici su pazienti con più di 45 anni la sua incidenza è compresa frail 3 e il $3,7 \%$.

Le manifestazioni cliniche del morbo di Paget dipendono dall'estensione e dalla localizzazione della malattia; la colonna vertebrale è una delle localizzazioni più tipiche di questa patologia (soprattutto il segmento lombare), così come il femore, la tibia e le ossa pelviche.

La sintomatologia dolorosa può essere causata da fratture locali, dall'aumento di dimensioni e di vascolarizzazione dell'osso o da compressione delle strutture nervose adiacenti al segmento interessato.

La calcemia, la calciuria e la fosforemia sono in genere nella norma, ad indicare un aumento simile di distruzione e di sintesi del tessuto osseo, mentre si nota un'aumentata concentrazione plasmatica delle idrossipiridinoline, delle lisilpiridinoline e di N-telopeptide. Particolare valore è da attribuire alla fosfatasi alcalina, i cui livelli sierici si correlano sia con l'estensione che con l'attività della malattia $[39,40]$.

Uno studio condotto da Adami e colleghi aveva come obiettivo quello di valutare l'efficacia di una terapia endovenosa di breve durata, costituita da 2 infusioni consecutive di neridronato ev in pazienti affetti da morbo di Paget in fase attiva. 83 pazienti sono stati randomizzati a ricevere $12.5,25,50 \mathrm{o} 100 \mathrm{mg}$ al giorno per due giorni consecutivi con complessive dosi totali rispettivamente di 25,50 , 100 e $200 \mathrm{mg}$ e un follow up di 180 giorni.

La variazione del livello sierico di fosfatasi alcalina, che durante il baseline era superiore alla norma almeno del $10 \%$, costituiva l'end point primario dello studio, unitamente a quella del suo isoenzima osseo e all'escrezione urinaria di N-telopeptide.

Tutte le dosi di neridronato hanno diminuito significativamente i marker biochimici di attività di malattia; in particolare, il nadir (la media dei tre valori più bassi registrati consecutivamente) dei livelli di fosfatasi alcalina totale era compreso tra $-16 \%$ e $-57,5 \%$ rispetto ai valori iniziali, con una significativa correlazione alla dose somministrata; correlazione riscontrabile anche per quanto riguarda la percentuale di pazienti che dopo sei mesi presentava ancora una risposta, sebbene parziale (Figura 6). 
La variazione della fosfatasi alcalina ossea e dell' escrezione di N-telopeptide, inoltre, è risultata correlata alla diminuzione del dolore osseo collegato alla patologia, significativamente diminuito nei responders biochimici [41]

Un altro studio, condotto per un periodo di 12 mesi su 32 pazienti affetti da morbo di Paget, ha valutato l'efficacia di $200 \mathrm{mg}$ di neridronato ev somministrato in singola dose, $\mathrm{o}$ in due dosi elargite in giorni consecutivi a pazienti che non erano mai stati trattati con agenti antiriassorbitivi $(n=15)$ e a pazienti che non avevano avuto risultati soddisfacenti tramite terapia con clodronato $(n=17)$. All'inizio della terapia non sono state rilevate differenze statisticamente significative nelle concentrazioni dei marker biologici assunti come indicatori del riassorbimento osseo (isoenzima osseo della fosfatasi alcalina, deossipiridinolina, peptide $\mathrm{N}$-terminale e $\mathrm{C}$-terminale del collageno di tipo 1) fra i pazienti precedentemente in terapia con clodronato e quelli mai trattati.

Dopo la prima somministrazione il neridronato ha indotto una diminuzione media percentuale dell'eccesso (definito come differenza tra i valori misurati e il punto medio del range di normalità) di isoenzima osseo della fosfatasi alcalina (bAp) circa del $68 \%$ e dell' eccesso di deossipiridinolina, di peptide N-terminale e di peptide $\mathrm{C}$-terminale rispettivamente $\mathrm{di}$ $68,1 \%, 60,6 \%$ e $86,7 \%$.

L'analisi ha rilevato che i marker di riassorbimento osseo diminuivano più lentamente nei pazienti che avevano assunto precedentemente clodronato, anche se l'entità della variazione appariva sostanzialmente uguale.

In 15 dei 21 pazienti che facevano uso di analgesici per controllare il dolore correlato alla patologia, si è verificato un miglioramento sintomatologico con un controllo completo del dolore in otto pazienti; 21 pazienti hanno manifestato remissione della malattia (rientro della bAp in un range di normalità), senza sostanziali differenze fra la somministrazione unica e quella in due fasi, e fra i pazienti precedentemente trattati e quelli non [42].

Questi dati confermano l'efficacia del neridronato nel trattare il morbo di Paget sia come bifosfonato di prima scelta, sia come terapia alternativa in caso di recidiva con clodronato.

Oltre ad essere potenti inibitori del riassorbimento osseo, alcuni bifosfonati hanno dimostrato di avere proprietà antinfiammatorie: in vitro, essi inibiscono la proliferazione linfocitaria, la sintesi delle prostaglandine [43-45] e l'enzima collagenasi-3 [46]. Ad esempio, in un trial controllato, la somministrazione di alendro- nato per tre mesi in pazienti affetti da artrite reumatoide (RA) ha diminuito considerevolmente i livelli sierici di IL-1, IL-6 e TNF- $\alpha$ [47]; in contrasto con questi dati, i risultati relativi ad uno studio svolto su 18 pazienti RA a cui e stato somministrato per via endovenosa pamidronato, un aminobifosfonato, non hanno rilevato nessun effetto antinfiammatorio.

In considerazione di questi dati contrastanti e del fatto che recentemente alcune analisi in vitro e in vivo $[48,49]$ hanno addirittura rilevato una possibile azione proflogistica da parte degli aminobifosfonati (probabilmente mediante stimolazione della sintesi di istamina e della maturazione di macrofagi e granulociti), è stato svolto uno studio al fine di valutare se il neridronato potesse avere effetti sul sistema immunitario e, se sì, di quale entità.

45 pazienti con artrite reumatoide attiva sono stati randomizzati in doppio cieco a ricevere una singola infusione di $25 \mathrm{mg}(\mathrm{n}=15)$ o di $50 \mathrm{mg}(\mathrm{n}=15)$ del farmaco in esame contro placebo $(\mathrm{n}=15)$.

Dopo 7 giorni dall'infusione, nel gruppo randomizzato a $25 \mathrm{mg}$, si è assistito ad un calo significativo della proteina $\mathrm{C}$ reattiva e della VES, mentre in nessuno dei tre gruppi si è verificata una variazione nell'indice articolare di Ritchie (i tre parametri assunti come indicatori dell'attività della malattia) [50].

Tali risultati suggeriscono che il neridronato possa modulare la flogosi in modo diverso a seconda della posologia con cui viene assunto; questo potrebbe rappresentare un fattore sfavorevole al suo utilizzo, anche se i risultati positivi ottenuti a livello di concentrazione di proteina $\mathrm{C}$ reattiva e velocità di eritrosedimentazione possono essere delle ottime basi per progettare studi più accurati volti a testare l'azione a lungo termine di questo farmaco.

Recentemente è stato segnalato che il neridronato, e gli aminobifosfonati in generale, potrebbero inibire la sintesi dello squalene e del colesterolo, al contrario dei bifosfonati non contenenti un gruppo amminico, che non interferiscono nei processi di sintesi degli steroli.

Per stimare la rilevanza di tale effetto, durante un trial volto a determinare gli effetti del neridronato sul riassorbimento osseo, sono stati monitorati i profili lipidici delle 87 pazienti incluse nello studio, randomizzate a ricevere $50 \mathrm{mg}$ ev del farmaco ogni due mesi $(\mathrm{n}=44)$ o placebo $(n=43)$. Il trattamento con neridronato ha determinato in queste pazienti una riduzione significativa nelle concentrazioni sieriche di colesterolo LDL (-4\% al secondo mese) e APO B (-6\%), e aumenti rilevanti nei valori di APO A-1 e di colesterolo HDL (+4\% al secon- 


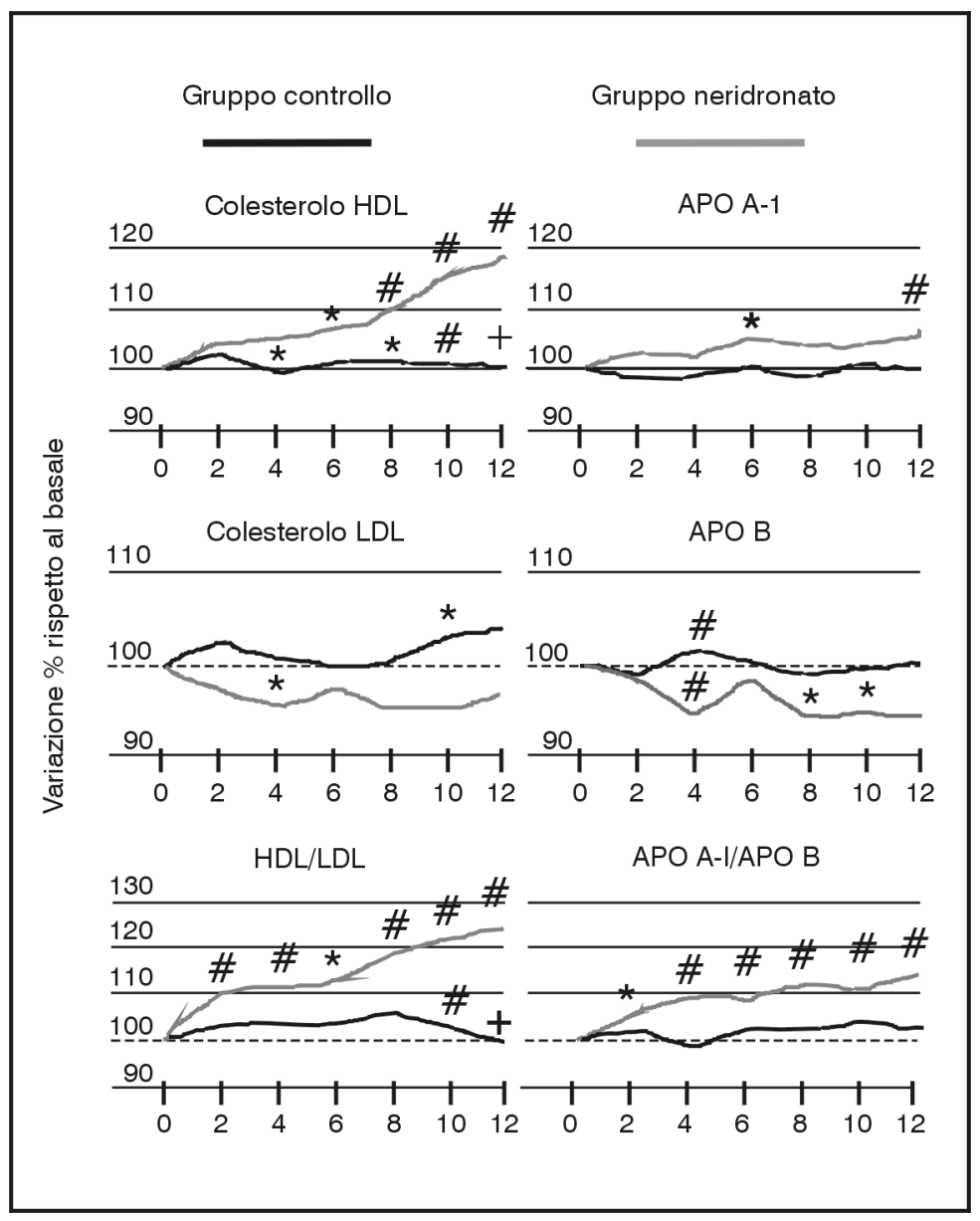

Figura 7

Variazioni \% dei valori sierici delle lipoproteine nel gruppo neridronato (linea grigia) rispetto al baseline. I simboli ( $\left.{ }^{*} p<0,05 ;{ }^{*} p<0,01 ;{ }^{+} p<0,001\right)$ sopra la linea grigia indicano significatività statistica del gruppo neridronato rispetto ai valori basali, mentre quelli sopra la linea nera (gruppo controllo) indicano la differenza tra gruppi imperfetta, 13 pazienti sui 31 randomizzati a neridronato hanno segnalato questo genere di reazione avversa 24-36 ore dopo la prima infusione ev, la cui durata non è stata comunque superiore alle 36 ore; di questi, quattro pazienti hanno riportato una reazione attenuata anche dopo la seconda infusione [24].

L'assunzione di neridronato ha causato una reazione di fase acuta (con dolore muscolare e febbre fino a $37,7^{\circ} \mathrm{C}$ ) anche nel $13 \%$ delle pazienti affette da osteoporosi post-menopausale esaminate da Braga e colleghi, percentuale che supera il $19 \%$ fra i pazienti pagetici sottoposti a terapia endovenosa a breve termine [41].

Durante lo studio condotto da Mazzantini e colleghi al fine di valutare i possibili effetti del farmaco sulla risposta infiammatoria, due pazienti $(n=15)$ dopo aver ricevuto $50 \mathrm{mg} \mathrm{di}$ neridronato ev hanno riportato un innalza-mento della temperatura, mentre nessuno dei pazienti randomizzati a ricevere $25 \mathrm{mg}$ del farmaco ha riportato reazioni di questo genere dimostrando un'apparente correlazione fra effetti indesiderati e dose somministrata [50].

Saltuariamente, durante il trattamento con questo farmaco, possono verificarsi anche disturbi elettrolitici, più frequentemente ipocalcemia e ipofosfatemia; per tale ragione, durante il trattamento con neridronato devono essere monitorati la funzionalità renale, nonché il calcio e il fosfato sierici, anche se nessuno dei pazienti partecipanti ai trial sopracitati ha riportato questo genere di effetto indesiderato. Più raramente il farmaco può portare a vertigini, cefalea, orticaria o ad altri rari effetti indesiderati fra cui disturbi ematici, quali leucopenia, e alterazioni degli enzimi epatici. La somministrazione per via intramuscolare è spesso accompagnata da dolore al sito di iniezione, che comunque si attenua dopo pochi minuti: disturbo riportato dal $45 \%$ dei pazienti partecipanti al trial condotto da Filipponi e colleghi [30].

In seguito alla somministrazione di bifosfonati recentemente è sorto un problema relativo alla comparsa di disturbi visivi, quali congiuntiviti, uveiti, scleriti, fotofobia, ecc. Un elevato numero di segnalazioni di questo tipo è stato riportato sul New England Journal of Medicine a carico di pamidronato, alendronato, etidronato, risedronato e clodronato, con una numerosità che riflette la loro diffusione di utilizzo.

Nel database del GIF (Gruppo Interregionale di Farmacovigilanza) sono presenti 10 segnalazioni di sospette reazioni avverse oculari non gravi da acido alendronico (6 nel 2003) con disturbi dell'accomodazione, visione of- 
fuscata, dolore oculare, tre segnalazioni da acido clodronico e due da acido risedronico (una irite nel 2003). Anche se nessun caso di reazioni simili è stato riportato a carico del neridronato, questo tipo di evento collaterale va sempre tenuto sotto controllo [52]. La reazione infiammatoria non pare in relazione alla dose, alla via di somministrazione o alla gravità della malattia che viene trattata.

\section{CONSIDERAZIONI FARMACOECONOMICHE}

In vista dei buoni risultati ottenuti in termini di efficacia nel trattamento dell' osteogenesi imperfetta e dietro sollecitazione di alcune associazioni di malati, il ministero della Salute italiano ha concesso l'autorizzazione al commercio del neridronato mediante le procedure preferenziali disposte per i farmaci orfani. Il farmaco è stato dunque collocato in fascia $\mathrm{A}$, senza note CUF, per la cura dell'osteogenesi imperfetta, prima che l'azienda produttrice terminasse la sperimentazione anche per l'osteoporosi.

Questa situazione ha generato un difetto di comunicazione tra ASL, medici e pazienti, in quanto alcuni specialisti, essendo a conoscenza dell' efficacia del farmaco su pazienti affetti da OI, lo prescrivono off label anche per il trattamento dell'osteoporosi.

Secondo il decreto-legge n. 23 del 17 febbraio 1998 (convertito in legge l'8 aprile 1998), in singoli casi il medico può, sotto la sua diretta responsabilità e previa informazione del paziente e acquisizione del consenso dello stesso, impiegare un medicinale prodotto industrialmente per un'indicazione o una via di somministrazione diversa da quella autorizzata. Secondo questo decreto, è lecito effettuare tale scelta qualora il medico ritenga, in base a dati documentabili, che il paziente non possa essere utilmente trattato con medicinali per $\mathrm{i}$ quali sia già approvata quella indicazione terapeutica (o quella via o modalità di somministrazione), e purché tale impiego sia noto e conforme a lavori apparsi su pubblicazioni scientifiche accreditate in campo internazionale. In nessun caso il ricorso del medico alla prescrizione off label può costituire riconoscimento del diritto del paziente alla erogazione dei medicinali a carico del Servizio sanitario nazionale, a meno che il farmaco non presenti caratteristiche particolari disciplinate dall' articolo 1, comma 4, del decreto-legge 21 ottobre 1996, n. 536.

Per il neridronato è quindi ammessa la rimborsabilità del farmaco soltanto per il trattamento dell'osteogenesi imperfetta; per tali ragioni focalizzeremo la nostra attenzione sulle conseguenze farmacoeconomiche derivanti dall'utilizzo del farmaco esclusivamente nel trattamento di tale patologia. Questo genere di analisi ha il fine di fornire al medico e agli altri decisori della spesa sanitaria gli strumenti adatti per scegliere razionalmente tra due o più strategie terapeutiche o tra una terapia e il "non trattamento"; ciò vale in particolar modo per quelle patologie come l'osteogenesi imperfetta che, pur non avendo un'incidenza elevata, sono malattie gravi, croniche e, di conseguenza, con un grande impatto sulla qualità della vita del paziente e dei suoi familiari.

La maggiore conoscenza delle sue basi genetiche e l'aumento delle possibilità di diagnosi, anche in casi precedentemente insospettabili, hanno indotto il passaggio dell'osteogenesi imperfetta dallo stato di malattia genetica "rara" a quello di uno dei disturbi genetici rilevati con maggior frequenza nella popolazione; anche se le forme più gravi sono clinicamente evidenti sin dalla nascita, forme più lievi di OI possono non essere sospettate sino a età avanzata [53].

Sulla base di queste considerazioni, si stima che attualmente negli Stati Uniti vi siano circa 50.000 individui affetti da OI, di cui il $60 \%$ con forme lievi; la forma letale più grave si verifica in circa 3-4 casi ogni 100.000 nascite. Le stime basate sulla presenza di fratture alla nascita variano da 1,6/100.000 a Singapore [54], 3,3/100.000 in Francia [55], e 15/100.000 nel Regno Unito [56], dati sottostimati a causa dei possibili aborti nei casi molto gravi, in cui pertanto la diagnosi alla nascita non è possibile; nell'insieme si stima l'esistenza di circa 0,5 milioni di persone con OI nel mondo $(0.008 \%)$.

In Italia ci sono circa 3.800 individui affetti da osteogenesi imperfetta in qualsiasi forma: la prevalenza è di circa 5 casi ogni 100.000 bambini nati [57]. Al momento sono disponibili tre tipi di trattamento: management non operativo (terapia fisica, riabilitazione, uso di busti ortopedici e apparecchi gessati), approccio chirurgico e approccio farmacologico. L'approccio chirurgico, previsto soprattutto nelle forme di OI più gravi, prevede principalmente il posizionamento intramidollare di chiodi e ha lo scopo di prevenire il rischio di fratture e garantire una migliore funzionalità degli arti inferiori. Questo tipo di intervento, sebbene produca un notevole miglioramento della capacità motoria e del conseguente livello di autonomia del paziente, comporta un elevato tasso di complicanze. Innanzitutto finché il paziente ha un'età in cui permane una significativa crescita ossea, risulta necessario infibulare l'osso con chiodi telescopici che, seguendo la cre- 
scita, possono essere tenuti in sede per un periodo più lungo rispetto ai chiodi non allungabili. Questo tipo di chiodi però richiedono interventi più complessi e sembrano aumentare il rischio di osteoporosi.

Le complicazioni più frequenti sono la migrazione del chiodo, spesso associata alla perforazione dell' articolazione, dell'osso e del tessuto molle periarticolare o la frattura stessa dell'osso infibulato; il rischio che si verifichino questi eventi è maggiore nei pazienti di età inferiore ai 5 anni e nelle tibie rispetto ai femori.

Dopo l'intervento è importante limitare l'immobilizzazione, per riprendere la riabilitazione il prima possibile; l'intervento, infatti, non è un fatto isolato ma è solo una tappa del cammino riabilitativo [58]

Nell'OI severa e moderatamente severa, i farmaci che agiscono favorendo la produzione di collagene tipo I, non rappresentano, in linea di principio, una terapia efficace.

Al momento una terapia farmacologica eziopatogenetica non esiste, mentre sono disponibili alcuni trattamenti sintomatici; in passato a tale scopo sono stati utilizzati gli steroidi anabolizzanti, la vitamina $\mathrm{D}$, la vitamina $\mathrm{C}$, il fluoruro di sodio, l'ossido di magnesio, i flavonoidi e la calcitonina, ma nessuno di questi ha mai dato buoni risultati nel lungo periodo.

Negli ultimi anni due sono i tipi di farmaci maggiormente usati nel trattamento dell'OI: i bifosfonati, di cui il neridronato è l'unico indicato per questa patologia, e l'ormone della crescita $(\mathrm{GH})$.

In letteratura si trovano pochi riferimenti relativi all'esperienza con GH soprattutto per quanto riguarda i trial controllati; dai dati analizzati si può concludere che l'ormone della crescita è una terapia utile nei pazienti con forme moderate di OI, sui quali ha dimostrato un'azione positiva sul turn over osseo, sulla densità minerale e sulla velocità di crescita; tuttavia i pazienti con scoliosi pre esistente o deformità ossee vanno trattati con particolare cautela visto il potenziale rischio di peggioramento di questi problemi [59]. I pazienti con OI grave non sembrano avere alcun vantaggio sostanziale da questa terapia [60-62]; inoltre, non sono noti i suoi effetti a lungo termine: il GH non ha ancora un'indicazione approvata per l'OI e i protocolli che lo utilizzano in associazione con altri farmaci nel trattamento di questa patologia sono al momento solo alla fase iniziale di sperimentazione.

Risultati più incoraggianti, come detto in precedenza, sono stati ottenuti con l'utilizzo dei bifosfonati e in particolar modo i derivati con un gruppo amino terminale: il neridronato ha dato ottimi risultati sia nei pazienti adulti che nel trattamento sintomatico dei bambini affetti da gravi forme di OI.

Per capire l'importanza di questi dati occorre soffermarsi sul notevole abbassamento della qualità di vita di un bambino affetto da OI e della sua famiglia. Nelle forme più gravi il bambino, ammesso che riesca a sopravvivere al parto, frequentemente nasce già con fratture ed è costretto a convivere costantemente con il rischio di andare incontro a deformità dovute all'incurvamento delle ossa, a corrosione dei denti e sordità e, ovviamente, a nuove fratture.

Ciò comporta tutta una serie di precauzioni che devono essere prese per evitare di esporre il paziente a ulteriori rischi, proteggendolo da situazioni che per le altre persone sono del tutto normali: il bambino affetto da OI difficilmente potrà frequentare la scuola, fare sport, giochi di gruppo o attività esterne, e spesso non sarà neanche in grado di deambulare senza il supporto di una sedia a rotelle, con tutti i problemi di discriminazione, di insicurezza e di sfiducia che ne derivano [63].

Utilizzare un farmaco in grado di ridurre, anche solo parzialmente, questi gravi disagi significa migliorare notevolmente la qualità di vita del bambino e dei suoi familiari. Per le famiglie che hanno un bambino affetto da OI la situazione descritta porta a conseguenze drastiche anche sul piano economico. Circa il $67 \%$ delle donne con un figlio disabile non ha la possibilità di trovare un lavoro o di mantenere quello che avevano prima della nascita del bambino [64], al contrario delle altre madri che, in seguito alla nascita del figlio, riescono a ritornare al lavoro nel $65 \%$ circa dei casi [65]. In media, questo tipo di introito rappresenta circa il 13\% del reddito totale della famiglia e, in Inghilterra, esso risulta determinante affinché circa un milione di nuclei familiari non scendano sotto la soglia di povertà.

Il motivo fondamentale per cui le madri di figli disabili sono impossibilitate a tornare al lavoro è certamente riscontrabile nel maggior bisogno di cure di cui essi necessitano [64].

Uno studio condotto in Gran Bretagna ha analizzato questa complicata situazione facendo un confronto fra le esperienze di famiglie con bambini disabili e quelle di famiglie con bambini non disabili. L'analisi è stata svolta mediante l'utilizzo di un "event diary" nel quale è stato chiesto alle famiglie di annotare tutte le cure dedicate al figlio, intendendo per cure tutte quelle azioni svolte al fine di preservare 
la sua salute, il suo benessere e la sua educazione. Inoltre alle famiglie è stato richiesto di compilare un questionario dettagliato sulla loro situazione finanziaria, sulla composizione del nucleo familiare, sugli aiuti che ricevevano da parenti, amici e istituzioni, sulle visite ospedaliere e sul tipo di diagnosi che era stata fatta al bambino (epilessia, autismo, osteogenesi imperfetta, ecc.); tale questionario è stato compilato con l'ausilio di un intervistatore incaricato della raccolta dei dati.

Dai risultati di questo studio appare chiaro che i bambini invalidi necessitano di un numero di cure per unità di tempo (waking hour) molto più elevato degli altri e soprattutto che, mentre per i bambini non disabili, questo numero decresce con l'età, per i bambini affetti da patologie gravi e croniche, il bisogno di sorveglianza e di protezione rimane costante.

Esso infatti dipende solo ed esclusivamente dalla gravità della malattia nel periodo considerato.

Delle 16 madri con figli disabili intervistate, 12 hanno dichiarato di non aver potuto riprendere il lavoro; questo, come già accennato, è una grave perdita a livello di budget familiare, ma il problema risulta ancora più serio se si considera che la maggior parte delle famiglie con disabili, per fronteggiare i costi delle cure, ha riportato un esborso di denaro molto maggiore rispetto a quelle con figli non disabili. L'ammontare di questa differenza di spesa è risultato in media di $£ 97,450$, delle quali $£$ 31,050 finanziate tramite sovvenzioni governative, $£ 26,000$ elargite da organizzazioni di beneficenza e $£ 40,400$ versate dalla famiglia stessa: come si può osservare quasi il $50 \%$ dell' ammontare resta a carico dei nuclei familiari, mettendo in grave difficoltà economica la maggior parte degli interessati [66].

In Italia l'osteogenesi imperfetta rientra nell'elenco delle malattie rare per le quali è riconosciuto il diritto all'esenzione dalla partecipazione al costo per le correlate prestazioni; l'assistito riconosciuto esente ha dunque diritto alle prestazioni, efficaci ed appropriate, incluse nei livelli essenziali di assistenza per il trattamento e il monitoraggio della malattia dalla quale è affetto e per la prevenzione degli ulteriori aggravamenti [67]. La qualità di vita per il paziente e per la sua famiglia, così come la disponibilità economica, restano tuttavia decisamente ridotte rispetto alla normalità: considerazione che sottolinea marcatamente la necessità di avere a disposizione una terapia in grado di migliorare il quadro clinico e sintomatico del paziente. Una confezione di neridronato costa 16,82 euro e contiene una fiala iniettabile da $25 \mathrm{mg}$.
La posologia orientativa è di $2 \mathrm{mg} / \mathrm{kg}$ di peso corporeo ogni tre mesi. Il costo per anno di terapia, assumendo che venga somministrata in tutti i casi la dose massima di $100 \mathrm{mg}$, risulta di 269,12 euro. Nel trial condotto da Adami et al [25], l'incidente di fratture registrate nei pazienti affetti da OI durante i due anni di terapia aveva dimostrato un calo significativo del $45 \%$ rispetto al numero di fratture verificatesi nei due anni precedenti l'inizio del trattamento.

Come si evince da questi dati, il costo del trattamento con neridronato risulta molto contenuto rispetto ai benefici derivanti dal suo utilizzo, soprattutto considerando che proprio la frattura rappresenta il maggior ostacolo che deve superare il paziente per potersi permettere una vita "normale".

\section{CONCLUSIONI}

Il neridronato è un aminobifosfonato di ultima generazione indicato nel trattamento dell' osteogenesi imperfetta, malattia rara e gravissima, per la cui indicazione è stato approvato con la procedura semplificata degli "orphan drugs".

La sua particolare formulazione lo rende adatto ad una somministrazione trimestrale per via endovenosa: ciò migliora notevolmente la compliance del paziente visto che i bifosfonati per via orale richiedono particolare attenzione nell'assunzione (sono scarsamente assorbiti) e comportano svariati effetti collaterali, soprattutto a livello gastrointestinale.

Dai trial effettuati, neridronato è risultato ben tollerato e molto efficace nell' aumentare la densità minerale ossea e nel diminuire il rischio di fratture nei pazienti affetti da osteogenesi imperfetta; inoltre la sua efficacia è stata testata con buoni risultati anche su pazienti affetti da osteoporosi post-menopausale e morbo di Paget. Dal punto di vista economico, il prezzo di acquisto del neridronato è tra $\mathrm{i}$ più bassi della sua classe; se paragonato ai benefici prodotti in termini di innalzamento della qualità di vita questo dato rivela l'efficienza farmacoeconomica del neridronato nella terapia dell'osteogenesi imperfetta. Infatti situazioni o movimenti che per una persona "normale" non rappresentano assolutamente un pericolo, per gli individui affetti dalla patologia possono diventare motivo di grave rischio: nelle forme più gravi la frequenza con cui avviene una frattura è elevatissima.

Si può facilmente dedurre quanto questo condizioni le normali attività del paziente, soprattutto perché una frattura rappresenta un evento traumatico sia dal punto di vista fisico 
che psicologico; inoltre spesso questo evento richiede ospedalizzazione, con conseguente aumento drastico delle risorse consumate e dei mancati guadagni da parte della famiglia o del paziente stesso, se adulto. Un farmaco che è in grado di diminuire sostanzialmente l'inci- denza di fratture e di preservare la massa ossea per un'età più avanzata può migliorare notevolmente la qualità di vita dei pazienti affetti da osteogenesi imperfetta, riducendone l'impatto clinico, sociale ed economico a carico delle famiglie e dell'intera società.

\section{BIBLIOGRAFIA}

1. Cole WG et al. Collagen genes: mutations affecting collagen structure and expression. Prog Nucleic Acid Res Mol Biol. 1994:47:29-80.

2. Paterson CR, McAllion S, Stellman JL. Osteogenesis imperfecta after the menopause. N Engl J Med. 1984 Jun 28;310(26):1694-6.

3. McAllion SJ, Paterson CR. Causes of death in osteogenesis imperfecta. J Clin Path 1996; 49: 627-30.

4. Shapiro F. Consequences of an osteogenesis imperfecta diagnosis for survival and ambulation. J Pediatr Orthop 1985; 5: 456-62.

5. Sillence DO, Senn A, Danks DM. Genetic heterogeneity in osteogenesis imperfecta. J Med Genet. 1979 Apr;16(2):10116.

6. Byers PH. Brittle bones-fragile molecules: disorders of collagen gene structure and expression. Trends Genet. 1990 Sep;6(9):293-300.

7. Hughes DE et al. Bisphosphonates promote apoptosis in murine osteoclasts in vitro and in vivo. J Bone Miner Res. 1995 Oct;10(10):1478-87.

8. Hughes DE et al. Inhibition of osteoclast-like cell formation by bisphosphonates in long-term cultures of human bone marrow. J Clin Invest. 1989 Jun;83(6):1930-5.

9. Sato M et al. Bisphosphonate action. Alendronate localization in rat bone and effects on osteoclast ultrastructure. J Clin Invest. 1991 Dec;88(6):2095-105.

10. Heaney RP. The bone-remodeling transient: implications for the interpretation of clinical studies of bone mass change. J Bone Miner Res. 1994 Oct;9(10):1515-23.

11. Compston JE. Prevention and management of osteoporosis. Current trends and future prospects. Drugs. 1997 May;53(5):727-35.

12. Tobias JH. How do bisphosphonates prevent fractures? Ann Rheum Dis. 1997 Sep;56(9):510-1.

13. Fleisch H. Bisphosphonates: mechanisms of action and clinical use in osteoporosis - an update. Horm Metab Res. 1997 Mar;29(3):145-50.

14. Gallini G, Cangini F. Ruolo dei mediatori dell'infiammazione nei meccanismi di regolazione e nelle interazioni tra cellule ossee- seconda parte. Il dentista moderno (aggiornamento monografico). 2003 Gen.

15. Sartori L et al. Injectable bisphosphonates in the treatment of postmenopausal osteoporosis. Aging Clin Exp Res. 2003 Aug;15(4):271-83.

16. Gotcher JE, Jee WS. The progress of the periodontal syndrome in the rice cat II. The effects of a diphosphonate on the periodontium. J Periodontal Res. 1981 Jul;16(4):441-55.

17. Li M et al. Parathyroid hormone monotherapy and cotherapy with antiresorptive agents restore vertebral bone mass and strength in aged ovariectomized rats. Bone. 1995 Jun;16(6):629-35.

18. Nerixia ${ }^{\circledR}$ - riassunto delle caratteristiche del prodotto.

19. Maconi G, Porro GB. Multiple ulcerative esophagitis caused by alendronate. Am J Gastroenterol. 1995 Oct;90(10):188990. 
20. Ettinger B, Pressman A, Schein J. Clinic visits and hospital admissions for care of acid-related upper gastrointestinal disorders in women using alendronate for osteoporosis. Am J Manag Care. 1998 Oct;4(10):1377-82.

21. Adami S, Zamberlan N. Adverse effects of bisphosphonates. A comparative review. Drug Saf. 1996 Mar;14(3):158-70.

22. Fleisch H. Bisphosphonates: mechanisms of action. Endocr Rev. 1998 Feb;19(1):80-100.

23. Kasting GB, Francis MD. Retention of etidronate in human, dog, and rat. J Bone Miner Res. 1992 May;7(5):513-22.

24. Adami et al. Intravenous neridronate in adults with osteogenesis imperfecta. J Bone Miner Res. 2003 Jan;18(1):12630.

25. Adami S et al. Intravenous neridronate In the treatment of patients with osteogenesis imperfecta. Sixth workshop on bisphosphonates from laboratory to the patient, Davos 2002.

26. Gatti D, Colapietro F, Adami S. bisfosfonati e osteogenesi imperfetta. Gibis - Rivista Bisfosfonati 2002 Giu; 3 (2):3-8.

27. XIX Convegno Nazionale AS.IT.O.I. Caserta 16-18 Maggio 2003. Sito ufficiale dell' Associazione Italiana Osteogenesi Imperfetta - www.asitoi.it.

28. Aki S et al. Gastrointestinal side effect profile due to the use of alendronate in the treatment of osteoporosis. Yonsei Med J. 2003 Dec 30;44(6):961-7.

29. Braga V et al. Intravenous intermittent neridronate in the treatment of postmenopausal osteoporosis. Bone. 2003 Sep;33(3):342-5.

30. Filipponi $\mathrm{P}$ et al. Two years neridronate increases bone mineral density in postmenopausal women affectes by osteoporosis. Bone 2002; 30 (Suppl 3): S48.

31. Wimalawqansa SJ. Intermittent intravenous pamidronate therapy: highly effective treatment for postmenopausal osteoporosis. J Bone Miner Res 2000; 16 (Suppl 1): S405.

32. Thiebaud D et al. Three monthly intravenous injections of ibandronate in the treatment of postmenopausal osteoporosis. Am J Med. 1997 Oct;103(4):298-307.

33. Adami S et al. Three-monthly $2 \mathrm{mg}$ intravenous Ibandronate injections restore bone turnover to premenopausal levels. J Bone Miner Res 2002; 17 (Suppl 1): S472.

34. Reid IR et al. Intravenous zoledronic acid in postmenopausal women with low bone mineral density. N Engl J Med. 2002 Feb 28;346(9):653-61.

35. Filipponi $\mathrm{P}$ et al. Cyclical clodronate is effective in preventing postmenopausal bone loss: a comparative study with transcutaneous hormone replacement therapy. J Bone Miner Res. 1995 May;10(5):697-703.

36. Heikkinen JE et al. Short-term intravenous bisphosphonates in prevention of postmenopausal bone loss. J Bone Miner Res. 1997 Jan;12(1):103-10.

37. Rossini M et al. Intramuscular clodronate therapy in postmenopausal osteoporosis. Bone. 1999 Feb;24(2):125-9.

38. Filipponi $\mathrm{P}$ et al. Intermittent versus continuous clodronate administration in postmenopausal women with low bone mass. Bone. 2000 Mar;26(3):269-74.

39. Singer FR, Krane SM. Paget's disease of bone. In Metabolic bone diseas. Accademic Press, 1998: 545-582.

40. Delmas PD, Meunier PJ. The management of Paget's disease of bone. N Engl J Med. 1997 Feb 20;336(8):558-66.

41. Adami S et al. Short-term intravenous therapy with Neridronate in Paget's disease. Clin Exp Rheumatol. 2002 JanFeb;20(1):55-8.

42. Filipponi $\mathrm{P}$ et al. Paget's disease of bone: benefits of neridonate as a first treatment and in cases of relapse after clodronate. Bone. 1998 Dec;23(6):543-8.

43. De Vries E et al. In vitro effect of (3-amino-1-hydroxypropylidene)-1,1-bisphosphonic acid (APD) on the function of mononuclear phagocytes in lymphocyte proliferation. Immunology. 1982 Sep;47(1):157-63.

44. Felix R, Bettex JD, Fleisch H. Effect of diphosphonates on the synthesis of prostaglandins in cultured calvaria cells. Calcif Tissue Int. 1981;33(5):549-52.

45. Ohya K et al. Effect of bisphosphonates on prostaglandin synthesis by rat bone cells and mouse calvaria in culture. Clin Sci (Lond). 1985 Oct;69(4):403-11.

46. Konttinen YT et al. Collagenase-3 (MMP-13) and its activators in rheumatoid arthritis: localization in the pannus-hard tissue junction and inhibition by alendronate. Matrix Biol. 1999 Aug;18(4):401-12. 
Neridronato nel trattamento dell'osteogenesi imperfetta: prestazioni cliniche ed economiche di un farmaco orfano

47. Cantatore FP, Acquista CA, Pipitone V. Evaluation of bone turnover and osteoclastic cytokines in early rheumatoid arthritis treated with alendronate. J Rheumatol. 1999 Nov;26(11):2318-23.

48. Richards PJ et al. Pro-inflammatory effects of the aminobisphosphonate ibandronate in vitro and in vivo. Rheumatology (Oxford). 1999 Oct;38(10):984-91.

49. Nakamura M et al. Contrast between effects of aminobisphosphonates and non-aminobisphosphonates on collageninduced arthritis in mice. Br J Pharmacol. 1996 Sep;119(2):205-12.

50. Mazzantini M et al. Single infusion of neridronate (6-amino-1-hydroxyhexylidene-1,1-bisphosphonate) in patients with active rheumatoid arthritis: effects on disease activity and bone resorption markers. Aging Clin Exp Res. 2002 Jun;14(3):197-201.

51. Adami S et al. Chronic intravenous aminobisphosphonate therapy increases high-density lipoprotein cholesterol and decreases low-density lipoprotein cholesterol. J Bone Miner Res. 2000 Mar;15(3):599-604.

52. Fraunfelder FW. Ocular side effects associated with bisphosphonates. Drugs Today (Barc). 2003 Nov;39(11):829-35.

53. Shapiro JR. Osteogenesi Imperfetta e altri difetti dello sviluppo osseo come cause occasionali di osteoporosi dell'adulto. Estratto da Osteoporosis. Second edition. Volume 2, capitolo 50.

54. Tan KL, Tock EP. Osteogenesis imperfecta congenita. Aust Paediatr J. 1971 Mar;7(1):49-53.

55. Robert JM et al. Hereditary bone fragility. Lyon Med. 1968 Mar 17;219(11):881-982.

56. Navani SV, Sarzin B. Intrauterine osteogenesis imperfecta. Review of the literature and a report of the radiological and necropsy findings in two cases. Br J Radiol. 1967 Jun;40(474):449-52.

57. Sito ufficiale di UILDM - Unione Italiana Lotta alla Distrofia Muscolare. www.uildm.org.

58. Osteogenesi Imperfetta: Linee Guida di Trattamento. Comitato scientifico dell' AS.IT.O.I - www.asitoi.it.

59. Antoniazzi F. Therapeutic approach with growth hormone. 7th International Conference on Osteogenesis Imperfecta. Montreal, Canada, 29 august - 1 september 1999.

60. Marini JC et al. The growth hormone and somatomedin axis in short children with osteogenesis imperfecta. J Clin Endocrinol Metab 1993; 76: 251-6.

61. Marini JC et al. Positive linear growth and bone responses to growth hormone treatment in children with types III and IV osteogenesis imperfecta: high predictive value of the carboxyterminal propeptide of type I procollagen. $\mathrm{J}$ Bone Miner Res 2003;18: 237-43.

62. Sillence D et al. Factors which influence the efficacy of growth hormone in 15 children with Osteogenesis Imperfecta types I and IV. Sixth International Conference on Osteogenesis Imperfecta. Utrecht, The Netherlands, 19-21 September 1996.

63. Rauch F, Glorieux FH. Osteogenesis imperfecta. Lancet. 2004Apr 24;363(9418):1377-85.

64. Dobson B, Middleton S. Paying to care: the cost of childhood disability. York, UK: Joseph Rowntree Foundation. 1998.

65. Lawton D. The family fund database. York, UK: University of York. 1996.

66. Curran AL et al. Time costs of caring for children with severe disabilities compared with caring for children without disabilities. Dev Med Child Neurol. 2001 Aug;43(8):529-33.

67. Sito del ministero della salute - www.ministerosalute.it. 